\title{
THE MONGE-AMPÈRE QUASI-METRIC STRUCTURE ADMITS A SOBOLEV INEQUALITY
}

\author{
Diego Maldonado
}

Abstract. Sobolev inequalities associated to the Monge-Ampère quasi-metric structure are proved.

\section{Introduction and main result}

The Monge-Ampère measure associated to a twice-differentiable convex function $\varphi$ : $\mathbb{R}^{n} \rightarrow \mathbb{R}$ is defined as $\mu_{\varphi}(x):=\operatorname{det} D^{2} \varphi(x)$. Given $x \in \mathbb{R}^{n}$ and $t>0$, a section of $\varphi$ centered at $x$ with height $t$ is the open bounded convex set

$$
S_{\varphi}(x, t):=\left\{y \in \mathbb{R}^{n}: \varphi(y)<\varphi(x)+\langle\nabla \varphi(x), y-x\rangle+t\right\} .
$$

The relevant compatibility condition between the sections of $\varphi$, which from now on we assume to be strictly convex, and its Monge-Ampère measure is the so-called $(D C)$ doubling condition. More precisely, we write $\mu_{\varphi} \in(\mathrm{DC})_{\varphi}$ if there exist constants $B \geq 1$ and $0<\alpha<1$ such that for all sections $S_{\varphi}(x, t)$

$$
\mu_{\varphi}\left(S_{\varphi}(x, t)\right) \leq B \mu_{\varphi}\left(\alpha S_{\varphi}(x, t)\right),
$$

where $\alpha S_{\varphi}(x, t)$ denotes $\alpha$-contraction of $S_{\varphi}(x, t)$ with respect to its (Euclidean) center of mass $x^{*}$. Constants depending only on $B$ and $\alpha$ in (1.2) as well as on dimension $n$ will be called geometric constants. The Monge-Ampère quasi-metric structure was introduced by Caffarelli and Gutiérrez [1] in their pioneering work on the linearized Monge-Ampère equation. Remarkably, the strictly convex function $\varphi$ generates a quasi-metric if and only if $\mu_{\varphi}$ possesses the $(D C)$-doubling property, (see, for instance, $[4$, Section 2]). More precisely, under the $(D C)$-doubling condition, $\varphi$ renders a structure of space of homogeneous type, see [4, Section 2] and references there in, in such a way that the function

$$
\rho_{\varphi}(x, y):=\varphi(y)-\varphi(x)-\langle\nabla \varphi(x), y-x\rangle, \quad x, y \in \mathbb{R}^{n},
$$

becomes a quasi-distance in $\mathbb{R}^{n}$, that is, $\rho_{\varphi}(x, y)=0$ if and only if $x=y ; \rho_{\varphi}(x, y) \simeq$ $\rho_{\varphi}(y, x)$; and $\rho_{\varphi}(x, y) \lesssim \rho_{\varphi}(x, z)+\rho_{\varphi}(z, y)$, where the implicit constants are geometric constants. By definition (1.1), the sections of $\varphi$ can then be realized as the quasi-balls associated to $\rho_{\varphi}$. On the other hand, if $\rho_{\varphi}$ generates a quasi-metric then the section of $\varphi$ satisfy the so-called engulfing property, which, in turn, is equivalent to $(D C)$, see $[2,3,6,7]$.

Received by the editors September 3, 2012.

2000 Mathematics Subject Classification. Primary 49N60; Secondary 35J60.

Key words and phrases. Sobolev inequalities, reverse-Hölder inequalities, linearized MongeAmpère operator. 
We will use the fact that the $(D C)$-doubling property implies the existence of geometric constants $K_{1}, K_{2}>0$ such that

$$
K_{1}^{n} t^{n} \leq\left|S_{\varphi}(x, t)\right| \mu_{\varphi}\left(S_{\varphi}(x, t)\right) \leq K_{2}^{n} t^{n}, \quad \forall x \in \mathbb{R}^{n}, \forall t>0 .
$$

For the proof this statement, as well as the real analysis associated to $\varphi$ and further characterizations of $(D C)$, see, for instance, [1], Theorem 8 in [2], Theorem 4 in [3], [7], and $[6$, Chapter 3].

Suppose that $\mu_{\varphi}(x)>0$ for a.e. $x \in \mathbb{R}^{n}$. The linearized Monge-Ampère operator, denoted by $L_{\varphi}$, is the typically degenerate, elliptic operator defined as

$$
L_{\varphi}(u)(x):=\operatorname{trace}\left(A_{\varphi}(x) D^{2} u(x)\right) \text { a.e. } x \in \mathbb{R}^{n},
$$

with

$$
A_{\varphi}(x):=\mu_{\varphi}(x)\left(D^{2} \varphi(x)\right)^{-1} \text { a.e. } x \in \mathbb{R}^{n} .
$$

The study of $L_{\varphi}$ is best carried out within the Monge-Ampère structure, see [1-4]. In [8], the author proved Poincaré-type inequalities for the Monge-Ampère quasimetric structure, which were instrumental in his proof of Harnack's inequality for non-negative solutions to $L_{\varphi}(u)=0$ (always under the hypothesis $\mu_{\varphi} \in(\mathrm{DC})_{\varphi}$ only).

In [9], Tian and Wang proved that if $\mu \in\left(\mu_{\infty}\right)$ (in their notation, $\mu \in(\mathbb{C} \mathbb{G}$ ), see [9]) and if the sections of $\varphi$ satisfy certain size conditions (see Lemma 3.3 in [9]), then a power-like decay of the distribution function of Green functions of $L_{\varphi}$ holds true. Consequently, a Sobolev inequality associated to $A_{\varphi}$ follows. Namely, there exists $p>1$ such that for every section $S:=S_{\varphi}(x, t)$ there is a $C>0$ such that whenever $u \in C_{0}^{1}(S)$ (that is, $u$ is continuously differentiable and compactly supported in $S$ ) we have

$$
\left(\frac{1}{\mu_{\varphi}(S)} \int_{S}|u|^{p} d \mu_{\varphi}\right)^{\frac{1}{p}} \leq C\left(\frac{1}{\mu_{\varphi}(S)} \int_{S}\left\langle A_{\varphi} \nabla u, \nabla u\right\rangle\right)^{\frac{1}{2}} .
$$

The importance and variety of the Sobolev inequalities (1.6) have been thoroughly stressed in [9].

Our main result is the Sobolev inequality (1.7) below, which we prove resorting to neither the $\left(\mu_{\infty}\right)$ condition nor apriori size condition on the sections of $\varphi$. The $\left(\mu_{\infty}\right)$ condition is significantly stronger than the $(D C)$-condition, the gap being comparable to that between $A_{\infty}$ Muckenhoupt weights and doubling weights, see [4, Section 3] for a thorough discussion and examples. Moreover, under the $(D C)$-doubling condition only, we can guarantee a (perhaps not optimal but) uniform value of $p$ in (1.6). We prove

Theorem 1. Assume $\mu_{\varphi} \in(\mathrm{DC})_{\varphi}$. Then, there exists a geometric constant $K_{6}>0$ such that for every section $S:=S_{\varphi}\left(x_{0}, t\right)$ and every $u \in C_{0}^{1}(S)$, we have

$$
\left(\frac{1}{\mu_{\varphi}(S)} \int_{S}|u(x)|^{\frac{2 n}{n-1}} d \mu_{\varphi}(x)\right)^{\frac{n-1}{2 n}} \leq K_{6} t^{\frac{1}{2}}\left(\frac{1}{\mu_{\varphi}(S)} \int_{S}\left|\nabla^{\varphi} u(x)\right|^{2} d \mu_{\varphi}(x)\right)^{\frac{1}{2}}
$$

where

$$
\nabla^{\varphi} u(x):=D^{2} \varphi(x)^{-\frac{1}{2}} \nabla u(x) \quad \forall x \in S .
$$

By means of a duality argument involving convex conjugates we also obtain the following Sobolev-type inequality, now with respect to Lebesgue measure. 
Theorem 2. Assume $\mu_{\varphi} \in(\mathrm{DC})_{\varphi}$. Then, there exists a geometric constant $K_{7}>0$ such that for every section $S:=S_{\varphi}\left(x_{0}, t\right)$ and every $u \in C_{0}^{1}(S)$, we have

$$
\left(\frac{1}{|S|} \int_{S}|u(x)|^{\frac{2 n}{n-1}} d x\right)^{\frac{n-1}{2 n}} \leq K_{7} t^{\frac{1}{2}}\left(\frac{1}{|S|} \int_{S}\left|\nabla^{\varphi} u(x)\right|^{2} d x\right)^{\frac{1}{2}} .
$$

\section{Estimates for Green functions}

The main step in the proof of Theorem 1 is a power-like decay estimate for the distribution function of Green functions of $L_{\varphi}$ on the sections of $\varphi$. Namely,

Theorem 3. Suppose $\mu_{\varphi} \in(\mathrm{DC})_{\varphi}$. There exists a geometric constant $K_{3} \geq 1$ such that for every section $S:=S_{\varphi}(x, t)$ and every $z \in S_{\varphi}(x, t / 2)$ we have

$$
\mu_{\varphi}\left(\left\{y \in S: g_{S}(z, y)>\lambda\right\}\right) \leq K_{3} \mu_{\varphi}(S)^{1-n^{\prime}} t^{n^{\prime}} \lambda^{-n^{\prime}}, \quad \forall \lambda>0 .
$$

Here $n^{\prime}=n /(n-1)$ and $g_{S}$ denotes the Green function of $L_{\varphi}$ in $S$.

Proof. First, assume that $x=0, \varphi(0)=0$, and $\nabla \varphi(0)=0$. With these assumptions we have $\varphi \geq 0$ and $z \in S_{\varphi}(0, t)$ if and only if $\varphi(z)<t$. Set $S:=S_{\varphi}(0, t)$ and $S_{1 / 2}:=S_{\varphi}(0, t / 2)$.

By the Aleksandrov-Bakelman-Pucci maximum principle (see [4, Lemma 8], [5, Theorem 9.1]), whenever $h$ is a solution to $L_{\varphi}(h)=H \mu_{\varphi}$ in $S$ with $h=0$ on $\partial S$, then

$$
\sup _{S}|h| \leq C_{1}|S|^{1 / n}\left(\int_{S}|H|^{n} d \mu_{\varphi}\right)^{1 / n},
$$

where $C_{1}$ depends only on the dimension $n$. On the other hand, note that the problem

$$
\left\{\begin{aligned}
L_{\varphi}(h) & =H \mu_{\varphi} & & \text { in } S \\
h & =0 & & \text { on } \partial S
\end{aligned}\right.
$$

is always solvable for $H \in L^{n}\left(S, d \mu_{\varphi}\right)$ with $h \in W_{l o c}^{2, n}(S) \cap C(\bar{S})$ because $L_{\varphi}$ has second-order continuous coefficients and $\varphi \in C^{2}$ with $D^{2} \varphi>0$ (see [5, Section 9.6]). That is, we make use of the fact that $\varphi \in C^{2}$ and consequently the eigenvalues of $\left(D^{2} \varphi(x)\right)^{-1}$ will be bounded and bounded away from zero on compact subsets of $\mathbb{R}^{n}$. Notice that we use this fact only to deduce the existence of solutions.

Again by the maximum principle, if $H \geq 0$ and $h$ solves (2.2), then $h<0$ (unless $H \equiv 0$, which trivially yields $h \equiv 0)$. Fix $z \in S$ and define $T_{z}$ by

$$
\begin{aligned}
T_{z}: L^{n}\left(S, \mu_{\varphi}\right) & \rightarrow \mathbb{R} \\
H & \mapsto-h(z)
\end{aligned}
$$

Then $T_{z}$ is a positive linear functional in $L^{n}\left(S, \mu_{\varphi}\right)^{*}$ and, from (2.1),

$$
\left\|T_{z}\right\|_{L^{n}\left(S, \mu_{\varphi}\right)^{*}} \leq C_{1}|S|^{1 / n} .
$$

By the Riesz representation theorem, there is a non-negative function $g_{S}(z, \cdot) \in$ $L^{n^{\prime}}\left(S, \mu_{\varphi}\right)$, where $n^{\prime}=\frac{n}{n-1}$, such that

$$
T_{z}(H)=\int_{S} g_{S}(z, y) H(y) d \mu_{\varphi}(y)
$$


and

$$
\left(\int_{S} g_{S}(z, y)^{n^{\prime}} d \mu_{\varphi}(y)\right)^{\frac{1}{n^{\prime}}} \leq C_{1}|S|^{1 / n} .
$$

That is, for every $z \in S$ and $h \in W_{l o c}^{2, n}(S) \cap C(\bar{S})$, with $h=0$ on $\partial S$, we have

$$
\begin{aligned}
-h(z) & =\int_{S} g_{S}(z, y) L_{\varphi}(h)(y) d y \\
& =\int_{S} g_{S}(z, y) \operatorname{trace}\left(\left(D^{2} \phi(y)\right)^{-1} D^{2} h(y)\right) \mu_{\varphi}(y) d y .
\end{aligned}
$$

In particular, setting $h:=\varphi-t$ in (2.4), for every $z \in S_{1 / 2}$ we obtain

$$
\begin{aligned}
\frac{t}{2} \leq t-\varphi(z) & =\int_{S} g_{S}(z, y) \operatorname{trace}\left(D^{2} \varphi(y)^{-1} D^{2} \varphi(y)\right) d \mu_{\varphi}(y) \\
& =n \int_{S} g_{S}(z, y) d \mu_{\varphi}(y) .
\end{aligned}
$$

Next, we prove that Green functions $g_{S}(z, \cdot)$ satisfy a reverse-Hölder inequality uniformly for $z \in S_{1 / 2}$. Fix $z \in S_{1 / 2}$, and use (2.3), (1.4), and (2.5) to write

$$
\begin{aligned}
\left(\frac{1}{\mu_{\varphi}(S)} \int_{S} g_{S}(z, y)^{n^{\prime}} d \mu_{\varphi}(y)\right)^{\frac{1}{n^{\prime}}} & \leq C_{1} \frac{\mu_{\varphi}(S)^{1 / n}|S|^{1 / n}}{\mu_{\varphi}(S)} \leq C_{1} K_{2} \frac{t}{\mu_{\varphi}(S)} \\
& \leq 2 C_{1} K_{2} n \frac{1}{\mu_{\varphi}(S)} \int_{S} g_{S}(z, y) d \mu_{\varphi}(y) .
\end{aligned}
$$

Then, from Chebyshev's inequality and (2.6), for $\lambda>0$ and always for $z \in S_{1 / 2}$, we have

$$
\begin{aligned}
& \mu_{\varphi}\left(\left\{y \in S: g_{S}(z, y)>\lambda\right\}\right) \\
& \quad \leq \frac{\mu_{\varphi}(S)}{\lambda^{n^{\prime}}} \frac{1}{\mu_{\varphi}(S)} \int_{S} g_{S}(z, y)^{n^{\prime}} d \mu_{\varphi}(y) \\
& \quad \leq \frac{\mu_{\varphi}(S)}{\lambda^{n^{\prime}}}\left(\frac{2 C_{1} K_{2} n}{\mu_{\varphi}(S)} \int_{S} g_{S}(z, y) d \mu_{\varphi}(y)\right)^{n^{\prime}} \\
& \quad=\frac{\left(2 C_{1} K_{2}\right)^{n^{\prime}} \mu_{\varphi}(S)^{1-n^{\prime}}(t-\varphi(z))^{n^{\prime}}}{\lambda^{n^{\prime}}} \\
& \leq\left(2 C_{1} K_{2}\right)^{n^{\prime}} \frac{\mu_{\varphi}(S)^{1-n^{\prime}} t^{n^{\prime}}}{\lambda^{n^{\prime}}}=: K_{3} \frac{\mu_{\varphi}(S)^{1-n^{\prime}} t^{n^{\prime}}}{\lambda^{n^{\prime}}},
\end{aligned}
$$

where we also used (2.5). For an arbitrary $x_{0} \in \mathbb{R}^{n}$ and a general section $S_{\varphi}\left(x_{0}, t\right)$, define

$$
\varphi_{x_{0}}(x):=\varphi\left(x_{0}-x\right)-\varphi\left(x_{0}\right)+\left\langle\nabla \varphi\left(x_{0}\right), x\right\rangle \quad \forall x \in \mathbb{R}^{n} .
$$

Then $\mu_{\varphi_{x_{0}}}$ verifies the $(D C)$-doubling property with the same constants as $\mu_{\varphi}$ does (uniformly in $x_{0}$ ), also $\nabla \varphi_{x_{0}}(0)=0, \varphi_{x_{0}}(0)=0$ and

$$
S_{\varphi_{x_{0}}}(0, t)=x_{0}-S_{\varphi}\left(x_{0}, t\right) .
$$

Thus, $\mu_{\varphi_{x_{0}}}\left(S_{\varphi_{x_{0}}}(0, t)\right)=\mu_{\varphi}\left(S_{\varphi}\left(x_{0}, t\right)\right)$ and if we now apply the obtained result to $\varphi_{x_{0}}$, the general case follows by changing variables $x_{0}-x \mapsto x$. 


\section{Proof of Theorem 1 via Tian-Wang's crucial lemma}

What follows is an adaptation, to the context of the Monge-Ampère structure, of the crucial lemma by Tian and Wang (see Lemma 2.1 in [9]). We include details of the proof, as well as some additions, for the sake of completeness and to follow up the geometric constants involved.

Let $S:=S_{\varphi}\left(x_{0}, t_{0}\right)$ be a section of $\varphi$ and let $G(z, y)$ denote the Green function of $L_{\varphi}$ on $2 S:=S_{\varphi}\left(x_{0}, 2 t_{0}\right)$. As before, let us assume that $x_{0}=0, \varphi(0)=0$, and $\nabla \varphi(0)=0$. By Theorem 3, for $z \in S$, we have

$$
\mu_{\varphi}(\{y \in 2 S: G(z, y)>\lambda\}) \leq K_{3} \mu_{\varphi}(2 S)^{1-n^{\prime}}\left(2 t_{0}\right)^{n^{\prime}} \lambda^{-n^{\prime}}, \quad \forall \lambda>0 .
$$

Let us set

$$
K:=K_{3} \mu_{\varphi}(2 S)^{1-n^{\prime}}\left(2 t_{0}\right)^{n^{\prime}}
$$

and $p:=2 n^{\prime}>2$ so that (3.1) reads as in Lemma 2.1 of [9], that is,

$$
\mu_{\varphi}(\{y \in 2 S: G(z, y)>\lambda\}) \leq K \lambda^{-\frac{p}{2}}, \quad \forall \lambda>0 .
$$

Next, consider any open set $U \subset S$ and let $\psi_{1}=\psi_{1}(U)$ and $\lambda_{1}=\lambda_{1}(U)$ be the first Dirichlet eigenfunction and eigenvalue of $L_{\varphi}$ in $U$, that is,

$$
\left\{\begin{aligned}
L_{\varphi}\left(\psi_{1}\right) & =-\lambda_{1} \psi_{1} \mu_{\varphi} & & \text { in } U \\
\psi_{1} & =0 & & \text { on } \partial U .
\end{aligned}\right.
$$

Let $G_{U}$ denote the Green function of $L_{\varphi}$ in $U$. Note that, since the coefficients of $A_{\varphi}$ are continuous and $A_{\varphi}$ is positive definite in $S, G_{U}$ always exists. By the maximum principle, $0 \leq G_{U}(z, y) \leq G(z, y)$ for every $z, y \in U(z \neq y)$.

Then $\psi_{1}$ and $G_{U}$ can be related by $\psi_{1}(y)=\lambda_{1} \int_{U} G_{U}(x, y) \mu_{\varphi}(x) d x$. Normalizing $\psi_{1}$ to be non-negative with $\left\|\psi_{1}\right\|_{L^{\infty}(\bar{U})}=1$ and taking $y_{1} \in U \subset S \subset 2 S$ such that $\psi_{1}\left(y_{1}\right)=1$, we can write

$$
1 \leq \lambda_{1}(U) \int_{U} G_{U}\left(x, y_{1}\right) \psi_{1}(x) \mu_{\varphi}(x) d x
$$

and then, for any $y_{0} \in U$, use (3.3) to estimate

$$
\begin{aligned}
\int_{U} G_{U}\left(x, y_{0}\right) \mu_{\varphi}(x) d x & =\int_{0}^{\infty} \mu_{\varphi}\left(\left\{x \in U: G_{U}\left(x, y_{0}\right)>\lambda\right\}\right) d \lambda \\
& \leq \int_{0}^{\infty} \min \left\{\mu_{\varphi}(U), \mu_{\varphi}\left(\left\{x \in U: G\left(x, y_{0}\right)>\lambda\right\}\right)\right\} d \lambda \\
& \leq \int_{0}^{\infty} \min \left\{\mu_{\varphi}(U), K \lambda^{-\frac{p}{2}}\right\} d \lambda=\tau \mu_{\varphi}(U)+K \int_{\tau}^{\infty} \lambda^{-\frac{p}{2}} d \lambda \\
& =n K^{\frac{2}{p}} \mu_{\varphi}(U)^{1-\frac{2}{p}},
\end{aligned}
$$

where $\tau:=\left(K / \mu_{\varphi}(U)\right)^{2 / p}$. Next, define $c^{*}:=\inf _{U \subset S} \lambda_{1}(U) \mu_{\varphi}(U)^{1-\frac{2}{p}}$, and note that $c^{*} \geq \frac{1}{n K^{2 / p}}>0$. Also define

$$
s^{*}:=\inf \left\{\int_{S}\left\langle A_{\varphi} \nabla u, \nabla u\right\rangle: u \in C_{0}^{1}(\bar{S}), \int_{S} F(u) \mu_{\varphi}=1\right\}
$$


where $F(u):=\int_{0}^{u} f(t) d t$ and, for a parameter $k>1$ to be sent to infinity,

$$
f(t):=\left\{\begin{aligned}
|t|^{p-1} & \text { if }|t|<k \\
k^{p-1} & \text { if }|t| \geq k
\end{aligned}\right.
$$

In order to make the relevant constants more explicit, we now complement the arguments in [9] by computing an upper bound for $s^{*}$. Since $S=S_{\varphi}\left(0, t_{0}\right)$, set

$$
h(x):=\left\{\begin{array}{cl}
\left(t_{0}-\varphi(x)\right)^{2} & \text { if } x \in S \\
0 & \text { if } x \in \mathbb{R}^{n} \backslash S .
\end{array}\right.
$$

Hence, by using the divergence theorem, the fact that

$$
\operatorname{div}\left(A_{\varphi}(x) \nabla u(x)\right)=\operatorname{trace}\left(A_{\varphi}(x) D^{2} u(x)\right), \quad \forall u \in C^{2},
$$

(since the columns of $A_{\varphi}$ are divergence free), and choosing $k>t_{0}^{2}$ (so that $0 \leq h=$ $|h| \leq t_{0}^{2}<k$ in $S$ ) in the definition of $f$ in (3.7), we obtain

$$
\begin{aligned}
\int_{S}\left\langle A_{\varphi}(x) \nabla h(x), \nabla h(x)\right\rangle d x= & -\int_{S} h(x) \operatorname{div}\left(A_{\varphi}(x) \nabla h(x)\right) d x \\
= & \int_{S} h(x) \operatorname{div}\left(2\left(t_{0}-\varphi(x)\right) A_{\varphi}(x) \nabla \varphi(x)\right) d x \\
= & 2 \int_{S} h(x)\left[\left(t_{0}-\varphi(x)\right) \operatorname{div}\left(A_{\varphi}(x) \nabla \varphi(x)\right)\right. \\
& \left.-\left\langle A_{\varphi}(x) \nabla \varphi(x), \nabla \varphi(x)\right\rangle\right] d x \\
\leq & 2 \int_{S} h(x)\left(t_{0}-\varphi(x)\right) \operatorname{div}\left(A_{\varphi}(x) \nabla \varphi(x)\right) d x \\
= & 2 \int_{S} h(x)\left(t_{0}-\varphi(x)\right) \operatorname{trace}\left(A_{\varphi}(x) D^{2} \varphi(x)\right) d x \\
= & 2 n \int_{S} h(x)\left(t_{0}-\varphi(x)\right) \mu_{\varphi}(x) d x=2 n \int_{S} h^{\frac{3}{2}}(x) \mu_{\varphi}(x) d x \\
\leq & 2 n\left(\int_{S} h^{p}(x) \mu_{\varphi}(x) d x\right)^{\frac{3}{2 p}} \mu_{\varphi}(S)^{\frac{2 p-3}{2 p}} \\
= & 2 n p^{\frac{3}{2 p}} \mu_{\varphi}(S)^{\frac{2 p-3}{2 p}}\left(\int_{S} F(h(x)) \mu_{\varphi}(x) d x\right)^{\frac{3}{2 p}},
\end{aligned}
$$

which implies

$$
s^{*} \leq 2 n p^{\frac{3}{2 p}} \mu_{\varphi}(S)^{\frac{2 p-3}{2 p}} .
$$

For a fixed $k$ (always large enough), let $v=v_{k} \in C^{1}(\bar{S})$ denote the function where the infimum (3.6) is attained. Therefore, $v$ satisfies

$$
\left\{\begin{aligned}
L_{\varphi}(v) & =-\hat{\lambda} f(v) \mu_{\varphi} & & \text { in } S \\
v & =0 & & \text { on } \partial S
\end{aligned}\right.
$$

Here $\hat{\lambda}$ is the Lagrange multiplier associated to the minimization. Take $x^{\prime} \in S$ such that

$$
v\left(x^{\prime}\right)=\|v\|_{L^{\infty}(S)}=: M .
$$


As in the proof of Lemma 2.1 in [9] we have

$$
\hat{\lambda} \leq s^{*} \leq p \hat{\lambda} .
$$

For $t \in(0, M)$ (here $M$ is as in (3.10)), set $\Omega_{t}:=\{x \in S: v(x)>M-t\}$. As in the proof of Lemma 2.1 in [9] one gets

$$
\mu_{\varphi}\left(\Omega_{t}\right) \geq \beta \mu_{\varphi}\left(\Omega_{t / 2}\right)^{\frac{p}{3 p-4}},
$$

where

$$
\beta:=\left(\frac{t c^{*}}{2 s^{*} M^{p-1}}\right)^{\frac{2 p}{3 p-4}} .
$$

By iteration of (3.12), for $m \in \mathbb{N}$ we have

$$
\mu_{\varphi}\left(\Omega_{t}\right) \geq \beta^{\sum_{k=0}^{m}\left(\frac{p}{3 p-4}\right)^{k}} \mu_{\varphi}\left(\Omega_{t / 2^{m}}\right)^{\left(\frac{p}{3 p-4}\right)^{m}} .
$$

The next step will be to show that

$$
\lim _{m \rightarrow \infty} \mu_{\varphi}\left(\Omega_{t / 2^{m}}\right)^{\left(\frac{p}{3 p-4}\right)^{m}}=1 .
$$

In order to show (3.15) we will now deviate from the proof of Lemma 2.1 in [9]. Indeed, instead of using a doubling property for $\mu_{\varphi}$ we use, yet again, that $\varphi \in C^{2}$ and $\mu_{\varphi}>0$ so that given a compact set $Q$ we have

$$
\mu_{\varphi}(x) \geq \inf _{Q} \mu_{\varphi}>0, \quad \forall x \in Q
$$

Continuing as in the proof of Lemma 2.1 in [9], set $a:=\|\nabla v\|_{L^{\infty}(S)}$, then

$$
\Omega_{t / 2^{m}} \supset B\left(x^{\prime}, t 2^{-m} / a\right),
$$

here $x^{\prime}$ is as in (3.10). Now, by (3.16) with $Q:=\bar{S} \operatorname{setting} \theta:=\inf _{Q} \operatorname{det} D^{2} \varphi>0$ we get

$$
\mu_{\varphi}\left(\Omega_{t / 2^{m}}\right) \geq \theta\left|B\left(x^{\prime}, t 2^{-m} / a\right)\right|=\theta \omega_{n}(t / a)^{n} 2^{-m n}, \quad \forall m \in \mathbb{N},
$$

where $\omega_{n}$ denotes the Lebesgue measure of the unit ball in $\mathbb{R}^{n}$. The bound (3.17) and the fact that $p>2$ then imply (3.15), and, consequently (from (3.14)),

$$
\mu_{\varphi}\left(\Omega_{t}\right) \geq \beta^{\frac{3 p-4}{2(p-2)}}=\left(\frac{t c^{*}}{2 s^{*} M^{p-1}}\right)^{\frac{p}{p-2}} .
$$

Note that in the proof of (3.15) no a priori rate of convergence as $m \rightarrow \infty$ is needed, so we were able to use (3.16) without resorting to any assumptions on a priori structural control of the infimum in (3.16). Also, since we are not using the hypothesis $\mu_{\varphi} \simeq 1$, we cannot follow the original reasoning in Lemma 2.1 in [9], because $\mu_{\varphi}$ is doubling on sections of $\varphi$, but not necessarily on Euclidean balls.

As in the proof of Lemma 2.1 in [9], setting $\eta:=k / M$ (and using (3.18)), we get

$$
\begin{aligned}
1 & \geq\left(\frac{c^{*}}{2 s^{*}}\right)^{\frac{p}{p-2}}\left[\int_{0}^{1-\eta} t^{\frac{p}{p-2}} \eta^{p-1} d t+p \int_{1-\eta}^{1} t^{\frac{p}{p-2}}(1-t)^{p-1} d t\right] \\
& =:\left(\frac{c^{*}}{2 s^{*}}\right)^{\frac{p}{p-2}} R(\eta) .
\end{aligned}
$$


Setting $\omega:=\{x \in S: v(x)>k\}$, as in the proof of Lemma 2.1 in [9] it follows that

$$
\mu_{\varphi}(\omega) \leq p k^{-p} .
$$

Also, on the set $\omega$ we have $L_{\varphi}(v)=-\hat{\lambda} k^{p-1} \mu_{\varphi}$, with $v=k$ on $\partial \omega$. Let $G_{\omega}$ denote the Green function of $L_{\varphi}$ in $\omega$ and let $x^{\prime}$ be as in (3.10). Hence, by (3.5), (3.20), (3.11), and (3.9)

$$
\begin{aligned}
M=v\left(x^{\prime}\right) & =k+\hat{\lambda} k^{p-1} \int_{\omega} G_{\omega}\left(x, x^{\prime}\right) \mu_{\varphi}(x) d x \leq k+2 \hat{\lambda} k^{p-1} K^{\frac{2}{p}} \mu_{\varphi}(\omega)^{1-\frac{2}{p}} \\
& \leq k+2 \hat{\lambda} k^{p-1} K^{\frac{2}{p}}\left(p k^{-p}\right)^{1-\frac{2}{p}}=\left(1+2 \hat{\lambda} K^{\frac{2}{p}}\right) k \\
& \leq\left(1+2 s^{*} K^{\frac{2}{p}}\right) k \leq\left(1+4 n p^{\frac{3}{2 p}} \mu_{\varphi}(S)^{\frac{2 p-3}{2 p}} K^{\frac{2}{p}}\right) k=: K_{4} k .
\end{aligned}
$$

Consequently, $\eta=\frac{k}{M} \geq \frac{1}{K_{4}}$ and, from (3.19)

$$
s^{*} \geq \frac{c^{*}}{2} \inf _{\eta \in\left[1 / K_{4}, 1\right]} R(\eta)^{\frac{p-2}{p}} \geq \frac{1}{4 K^{2 / p}} \inf _{\eta \in\left[1 / K_{4}, 1\right]} R(\eta)^{\frac{p-2}{p}}=: K_{5}>0 .
$$

At this point, given $u \in C_{0}^{1}(\bar{S})$, we can take limits as $k \rightarrow \infty$ to obtain

$$
\left(\int_{S} u^{p} d \mu_{\varphi}\right)^{\frac{1}{p}} \leq \frac{1}{K_{5}^{1 / 2}}\left(\int_{S}\left\langle A_{\varphi} \nabla u, \nabla u\right\rangle d x\right)^{\frac{1}{2}} .
$$

The seemingly awkward dependence of $K$ and $K_{5}$ on $\mu_{\varphi}(S)$ and $t_{0}$ can be circumvented by employing the normalization technique of Caffarelli and Gutiérrez [1]. Indeed, given a section $S=S_{\varphi}\left(x_{0}, t_{0}\right)$ let $T$ be an affine transformation normalizing $S$ so that, in the notation of $[1$, Section 1$]$, we have

$$
\begin{gathered}
\psi_{\lambda}(y):=\frac{1}{\lambda} \varphi\left(T^{-1} y\right), \quad B(0,1) \subset S^{*}:=T(S) \subset B(0, n) \\
\bar{\mu}(y)=\mu_{\Psi_{\lambda}}(y)=\frac{1}{\lambda^{n}}|T|^{-2} \mu_{\varphi}\left(T^{-1} y\right), \quad D^{2} \Psi_{\lambda}(y)=\frac{1}{\lambda}\left(T^{-1}\right)^{t} D^{2} \varphi\left(T^{-1} y\right) T^{-1}
\end{gathered}
$$

and $\mu_{\psi_{\lambda}}\left(S^{*}\right)=1$ so that

$$
\lambda^{n}|T|=\mu_{\varphi}\left(S_{\varphi}\left(x_{0}, t_{0}\right)\right) .
$$

Applying the previous proof to $S^{*}$ and $\Psi_{\lambda}$ and using the fact that

$$
c_{1} \leq \frac{t_{0}}{\lambda} \leq c_{2}
$$

for geometric constants $c_{1}$ and $c_{2}$ (see Theorem 8 in [2]), the constants $K$ in (3.2) and $K_{5}$ are now completely geometric (in particular, they are independent of $x_{0}$ and $t_{0}$ ). Consequently, for $\bar{u} \in C_{0}^{1}\left(\overline{S^{*}}\right)$, it follows that

$$
\left(\int_{S^{*}} \bar{u}(y)^{p} \bar{\mu}(y) d y\right)^{\frac{1}{p}} \leq \frac{1}{K_{5}^{1 / 2}}\left(\int_{S^{*}}\left\langle A_{\Psi_{\lambda}}(y) \nabla \bar{u}(y), \nabla \bar{u}(y)\right\rangle d y\right)^{\frac{1}{2}} .
$$

Finally, given $u \in C_{0}^{1}(\bar{S})$ we set $y=T x$ and $u(x):=\bar{u}(T x)$ for $x \in S_{\varphi}\left(x_{0}, t_{0}\right)$, then changing variables in (3.26) by means of (3.22)-(3.25), the Sobolev inequality (1.7) follows. For further considerations on the smoothness assumptions for $\varphi$, see Remark 3.2 in [9]. 


\section{Proof of Theorem 2 via convex conjugation}

Given a strictly convex, twice continuously differentiable $\varphi: \mathbb{R}^{n} \rightarrow \mathbb{R}$, its Legendre transform or convex conjugate, will be denoted by $\psi$. Under the hypothesis $\mu_{\varphi} \in$ $(\mathrm{DC})_{\varphi}$ we have that $\mu_{\psi}:=\operatorname{det} D^{2} \psi \in(\mathrm{DC})_{\psi}$ (with respect to the sections of $\psi$ ), where the $(\mathrm{DC})_{\psi}$-doubling constants for $\mu_{\psi}$ depend only on the ones for $\mu_{\varphi}$ and dimension $n$. Also, $\psi$ is a strictly convex twice continuously differentiable function whose domain is $\mathbb{R}^{n}$ and

$$
\nabla \varphi(\nabla \psi(x))=\nabla \psi(\nabla \varphi(x))=x \quad \forall x \in \mathbb{R}^{n},
$$

(see [3, Section 5]) which implies that

$$
D^{2} \varphi(\nabla \psi(y)) D^{2} \psi(y)=D^{2} \psi(\nabla \varphi(x)) D^{2} \varphi(x)=I \quad \forall x, y \in \mathbb{R}^{n}
$$

and that, for every Borel set $E \subset \mathbb{R}^{n}$,

$$
|E|=|\nabla \varphi(\nabla \psi(E))|=\mu_{\varphi}(\nabla \psi(E))=\mu_{\psi}(\nabla \varphi(E)) .
$$

Moreover, from Theorem 12 in [3], there exists a geometric constant $K_{0} \geq 1$ such that for every $x \in \mathbb{R}^{n}$ and $t>0$,

$$
\nabla \varphi\left(S_{\varphi}\left(x, t / K_{0}\right)\right) \subset S_{\psi}(\nabla \varphi(x), t) \subset \nabla \varphi\left(S_{\varphi}\left(x, K_{0} t\right)\right) .
$$

Next, given a section $S:=S_{\varphi}\left(x_{0}, t\right)$ of $\varphi$ set $y_{0}:=\nabla \varphi\left(x_{0}\right), S_{\psi}:=S_{\psi}\left(y_{0}, K_{0} t\right)$ and $S^{\varphi}:=\nabla \psi\left(S_{\psi}\right)$. By (4.4) we have that

$$
S=S_{\varphi}\left(x_{0}, t\right) \subset \nabla \psi\left(S_{\psi}\left(y_{0}, K_{0} t\right)\right)=S^{\varphi} .
$$

Given $u \in C_{0}^{1}(S)$, for $y \in S_{\psi}$ let us define $v(y):=u(\nabla \psi(y))$. Then, $\operatorname{supp}(v)=$ $\nabla \varphi(\operatorname{supp}(u)) \subset \nabla \varphi(S) \subset S_{\psi}$, hence $v \in C_{0}^{1}\left(S_{\psi}\right)$. By applying (1.7) (with respect to $\psi)$ to $v$ on $S_{\psi}$ we obtain

$$
\begin{aligned}
& \left(\frac{1}{\mu_{\psi}\left(S_{\psi}\right)} \int_{S_{\psi}}|v(y)|^{\frac{2 n}{n-1}} d \mu_{\psi}(y)\right)^{\frac{n-1}{2 n}} \\
& \quad \leq K_{6}^{\psi}\left(K_{0} t\right)^{\frac{1}{2}}\left(\frac{1}{\mu_{\psi}\left(S_{\psi}\right)} \int_{S_{\psi}}\left|\nabla^{\psi} v(y)\right|^{2} d \mu_{\psi}(y)\right)^{\frac{1}{2}} .
\end{aligned}
$$

By changing variables $y=\nabla \varphi(x),(4.1),(4.2)$, and (4.3) yield

$$
\begin{aligned}
\left(\frac{1}{\left|S^{\varphi}\right|} \int_{S^{\varphi}}|u(x)|^{\frac{2 n}{n-1}} d x\right)^{\frac{n-1}{2 n}} & \leq K_{6}^{\psi}\left(K_{0} t\right)^{\frac{1}{2}}\left(\frac{1}{\left|S^{\varphi}\right|} \int_{S^{\varphi}}\left|\nabla^{\varphi} u(x)\right|^{2} d x\right)^{\frac{1}{2}} \\
& \leq K_{6}^{\psi}\left(K_{0} t\right)^{\frac{1}{2}}\left(\frac{1}{|S|} \int_{S}\left|\nabla^{\varphi} u(x)\right|^{2} d x\right)^{\frac{1}{2}}
\end{aligned}
$$

where the last inequality is due to the fact that $u$ (and, therefore, $\nabla^{\varphi} u$ ) is supported in $S$ and $S \subset S^{\varphi}$. By Lemma 5.2(a) in [1], Lebesgue measure is doubling, with uniform constant $2^{n}$, with respect to the sections of any convex function. Then,

$$
\left|S_{\varphi}(x, t)\right| \leq 2^{n}\left|S_{\varphi}(x, t / 2)\right| \quad \forall x \in \mathbb{R}^{n}, \forall t>0 .
$$

In particular, recalling (4.5),

$$
|S| \leq\left|S^{\varphi}\right| \leq\left|S_{\varphi}\left(x_{0}, K_{0}^{2} t\right)\right| \leq\left(2 K_{0}^{2}\right)^{n}\left|S_{\varphi}\left(x_{0}, t\right)\right|=\left(2 K_{0}^{2}\right)^{n}|S| .
$$


Therefore,

$$
\begin{aligned}
& \left(\frac{1}{|S|} \int_{S}|u(x)|^{\frac{2 n}{n-1}} d x\right)^{\frac{n-1}{2 n}} \leq\left(\frac{\left(2 K_{0}^{2}\right)^{n}}{\left|S^{\varphi}\right|} \int_{S^{\varphi}}|u(x)|^{\frac{2 n}{n-1}} d x\right)^{\frac{n-1}{2 n}} \\
& \quad \leq K_{6}^{\psi}\left(2 K_{0}^{2}\right)^{\frac{(n-1)}{2}}\left(K_{0} t\right)^{\frac{1}{2}}\left(\frac{1}{|S|} \int_{S}\left|\nabla^{\varphi} u(x)\right|^{2} d x\right)^{\frac{1}{2}}
\end{aligned}
$$

and (1.9) follows with $K_{7}:=2^{\frac{(n-1)}{2}} K_{6}^{\psi} K_{0}^{n-\frac{1}{2}}$.

\section{Acknowledgments}

The author would like to thank Professor Truyen Nguyen for his careful reading of the manuscript and for pointing out an error in its initial draft and the anonymous referees for their helpful comments.

\section{References}

[1] L. Caffarelli and C. Gutiérrez, Properties of the solutions of the linearized Monge-Ampère equation, Amer. J. Math. 119(2) (1997), 423-465.

[2] L. Forzani and D. Maldonado, On geometric characterizations for Monge-Ampère doubling measures, J. Math. Anal. Appl. 275(2) (2002), 721-732.

[3] — - Properties of the solutions to the Monge-Ampère equation, Nonlinear Anal. 57(5-6) (2004), 815-829.

[4] $\longrightarrow$ A mean-value inequality for non-negative solutions to the linearized Monge-Ampère equation, Potential Anal. 30(3) (2009), 251-270.

[5] D. Gilbarg and M. Trudinger, Elliptic partial differential equations of second order, SpringerVerlag, Berlin-Heidelberg-New York, 2001.

[6] C. Gutiérrez, The Monge-Ampère equation, progress in nonlinear differential equations and their applications, 44, Birkhauser, 2001.

[7] C. Gutiérrez and Q. Huang, Geometric properties of the sections of solutions to the MongeAmpère equation, Trans. Amer. Math. Soc. 352(9) (2000), 4381-4396.

[8] D. Maldonado, On the $W^{2,1+\varepsilon}$-estimates for the Monge-Ampère equation and related real analysis, Calc. Var. Partial Differ. Equ. DOI is 10.1007/s00526-013-0629-1 and it appears online at http://link.springer.com/article/10.1007/s00526-013-0629-1

[9] G. Tian and X.-J. Wang, A class of Sobolev type inequalities, Methods Appl. Anal. 15 (2008), 257-270.

Kansas State University, Department of Mathematics, 138 Cardwell Hall, Manhattan, KS 66506, USA

E-mail address: dmaldona@math.ksu.edu 